\title{
Onset of action and seizure control in Lennox- Gaustaut syndrome: focus on rufinamide
}

\author{
Russell P Saneto' \\ Gail D Anderson ${ }^{2}$ \\ 'Division of Pediatric Neurology, \\ Seattle Children's Hospital/University \\ of Washington, Seattle, Washington, \\ USA; '2Department of Pharmacy, \\ University of Washington, Seattle, \\ Washington, USA
}

\begin{abstract}
Lennox-Gaustaut syndrome is an electroclinical epilepsy syndrome characterized by the triad of electroencephalogram showing diffuse slow spike-and-wave discharges and paroxysmal fast activity, multiple intractable seizure types, and cognitive impairment. The intractability to seizure medications and cognitive impairment gives rise to eventual institutionalized patient care. Only a small subset of seizure medications has been shown to be helpful in seizure control. Most patients take up to 3 medications at high therapeutic dosing and are susceptible to medication-induced side effects. The lack of medication efficacy in seizure control has led one meta-analysis to conclude that there is no single medication that is highly efficacious in controlling seizures in this syndrome. On this background, a new and structurally novel seizure medication, rufinamide, has been found to be beneficial in the treatment of seizures in this syndrome. In a multicenter, double-blinded, randomized, placebo-controlled study, rufinamide was found to reduce seizures by over 30\%. More importantly, it reduced the frequency of the seizure type that induces most of the morbidity of this syndrome, the drop seizure, by over $40 \%$. There were few side effects, the medication was well tolerated, and in the open labeled extension study, tolerance was not found. In this review, we describe the main electroclinical features of Lennox-Gaustaut syndrome and summarize the few controlled studies that have contributed to its rational treatment. Currently, there is no single agent or combination of agents that effectively treat the multiple seizure types and co-morbidities in this syndrome. Our focus will be on the role of the new medication rufinamide in seizure reduction in patients with Lennox-Gaustaut syndrome.
\end{abstract}

Keywords: Lennox-Gaustaut syndrome, catastrophic pediatric epilepsy, rufinamide

\section{Introduction}

The Lennox-Gaustaut syndrome is a catastrophic pediatric syndrome characterized by the electroclinical features of (1) electroencephalogram (EEG) showing abnormal background, diffuse slow spike-and-wave complexes (1.5-2.5 Hertz) and paroxysmal fast rhythms (10 Hertz), the latter may only occur in sleep; (2) multiple types of epileptic seizures typically including tonic, typical absence, and drop attacks; and (3) slow mental development and/or behavioral disturbance. ${ }^{1-3}$ The long-term prognosis is poor because the majority of patients are medically refractory to antiseizure drugs (AEDs), have frequent episodes of status epilepticus, and severe cognitive impairment. Due to these factors, most patients eventually required institutional care.

Treatment is unsatisfactory. The finding of multiple seizure types, and exacerbation of certain seizures by the very medication used to stop other seizure types, often leads to over-dosing of medication, ineffective treatment, and pronounced expression of side effects. Many, if not all patients, are on polytherapy. Only a small subset of AEDs has found to be efficacious in treatment of seizures associated with Lennox-Gaustaut syndrome. Even using these seizure medications, up to $96 \%$ of patients with LennoxGaustaut syndrome have seizures that are refractory to treatment. ${ }^{4,5}$ 
Against this background of partially effective treatments, a recent double-blind, randomized, placebo-controlled study found that a new seizure medication, rufinamide, reduced overall seizure frequency and in particular, tonic-atonic seizures in Lennox-Gaustaut syndrome. ${ }^{6}$ Based on the data derived from this study, the United States Food and Drug Administration (FDA) approved rufinamide for adjunctive treatment in patients with Lennox-Gaustaut syndrome in late 2008. Rufinamide has been approved in Europe since early 2007 for add-on treatment in this syndrome.

In this review, we will discuss the natural history and management of the electroclinical syndrome of LennoxGaustaut. In particular, our review will concentrate on the pharmacological properties and use of rufinamide in the context of the current knowledge of treatment for this medically intractable condition.

\section{Lennox-Gaustaut syndrome}

Lennox-Gaustaut syndrome is a catastrophic epileptic encephalopathy of childhood. Gibbs et $\mathrm{al}^{7}$ first described the EEG pattern of slow spike and wave with Lennox, and his colleagues Lennox and Davis, ${ }^{8}$ together with Dravet ${ }^{9}$ and Gaustaut et $\mathrm{l}^{10}$ further refined the clinical picture of the early onset of multiple seizure types and mental deficiency. Although this syndrome has a long history in the epilepsy literature, there is no consensus of what minimal features are required for diagnosis when one of the triad of EEG or clinical findings is missing. The definition is problematic as all features may not be present at a given period of the syndrome and the slow spike-and-wave pattern may be seen in other epileptic encephalopathies. Most, however, require two absolute criteria of (1) interictal, diffuse slow-spike-and-wave discharges on the EEG and (2) frequent and multiple seizure types, including tonic, atypical absences, and drop attacks together with supporting findings such as cognitive impairment, medically refractory seizures and paroxysmal fast activity in sleep. ${ }^{4,11}$

\section{Electroclinical features}

The age of onset is usually in early childhood between the ages of 1 and 8 years with peak at 3 to 5 years of age. ${ }^{12}$ Onset can vary, as some patients do not express their first seizure until the second decade. ${ }^{13}$ Those with Down's syndrome usually do not manifest the full syndrome until about 10 years of age. ${ }^{14}$ This syndrome accounts for approximately $1 \%$ to $10 \%$ of all childhood epilepsies. ${ }^{4,15}$ However, the real figure is likely $\leq 4 \%$ as most patients with Lennox-Gaustaut syndrome are seen in tertiary epilepsy centers accounting for the higher estimates of incidence. ${ }^{16,17}$
The etiology of the Lennox-Gaustaut syndrome is diverse with both cryptogenic and symptomatic causes. Symptomatic cases are due to a variety of diffuse gray matter lesions such as hypoxic-ischemia, infection, malformation of cortical development, chromosomal abnormalities, and head trauma. ${ }^{18}$ Cryptogenic etiology accounts for approximately $25 \%$ to $33 \%$ of patients. ${ }^{4,18,19}$ Lennox-Gaustaut syndrome can follow other epileptic encephalopathy syndromes, in particular, West syndrome and Ohthara syndrome..$^{20,21}$ In some studies, up to $25 \%$ of all patients have a history of West syndrome. ${ }^{18,15,20}$ The progression of EEG findings and multiple seizure types from hypsarrhythmia, suppression-bursts, and epileptic spasms suggests age-related factors in the evolution of the electroclinical syndrome of Lennox-Gaustaut.

The characteristic interictal EEG pattern of slow spikeand-wave complex (Figure 1) was initially described by Gibbs et al. ${ }^{7}$ The record is dominated by 1.5 to 2.5 Hertz spike/ sharp-and-wave complex, which are diffuse and maximum bifrontally but sometimes can be confined to the anterior or posterior head regions. Although the slow spike-and-wave pattern is fairly symmetrical, a shifting asymmetry in different bursts is common. ${ }^{18}$ The duration of the complexes can vary, but they often appear in prolonged sequences as long as half of the routine EEG record without clinical change. For this reason, the slow spike-and-wave activity is usually considered an interictal pattern. The slow spike-and-wave complexes are sometimes not present on the EEG at the onset of seizures, but can appear over 1 year later. ${ }^{22}$ Non-rapid eye movement sleep augments slow spike-and-wave complex expression in about $95 \%$ of patients with longer duration of discharges. ${ }^{18}$

Paroxysmal fast activity (Figure 2) is the second characteristic EEG pattern of Lennox-Gaustaut syndrome and occurs predominantly or sometimes exclusively during nonrapid eye movement sleep. ${ }^{15}$ Discharge frequency is usually between 10 and $25 \mathrm{Hertz}$, preceded or followed by generalized sharp and slow wave complexes, and has a duration of 1 to 9 seconds. The spike paroxysms show little change in frequency throughout the burst. The discharges are widespread in distribution and bilaterally synchronous over both hemispheres. Shifting asymmetries are common. When seen during the waking state, paroxysmal fast activity is usually ictal and associated with tonic seizures. However, in its most common finding during nonrapid eye movement sleep, the EEG pattern is usually not associated with obvious clinical signs. When not associated with clinical signs, this pattern may sometimes be subclinical seizures.

The background frequency is slow for age in $65 \%$ to $90 \%$ of patients. ${ }^{22}$ The degree of slowing correlates with the 


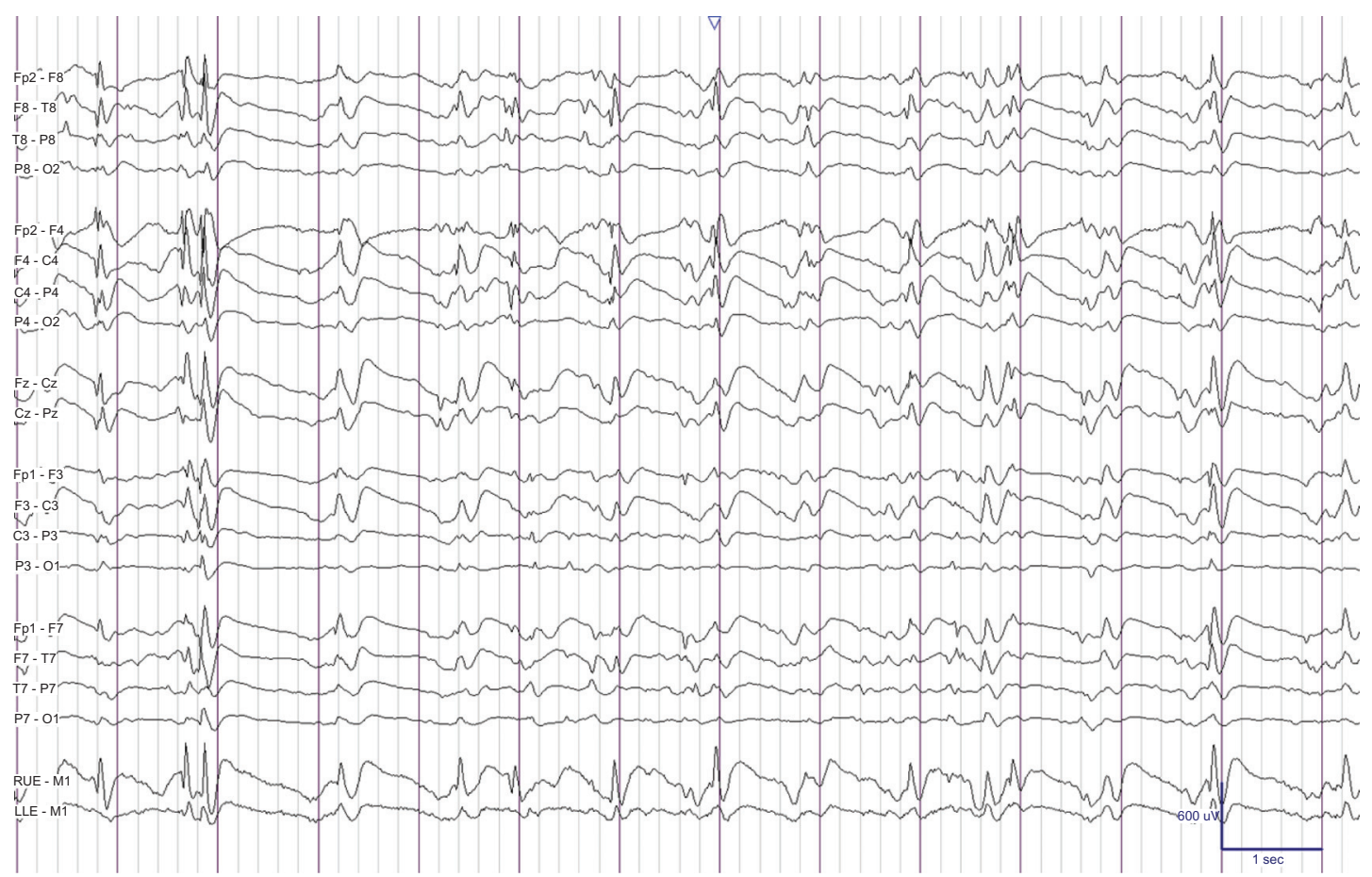

Figure I This is an EEG of a 5-year-old girl with Lennox-Gaustaut syndrome showing generalized spike-and-wave discharges. The frequency of spike-and-wave activity is $<2.5 \mathrm{Hertz}$

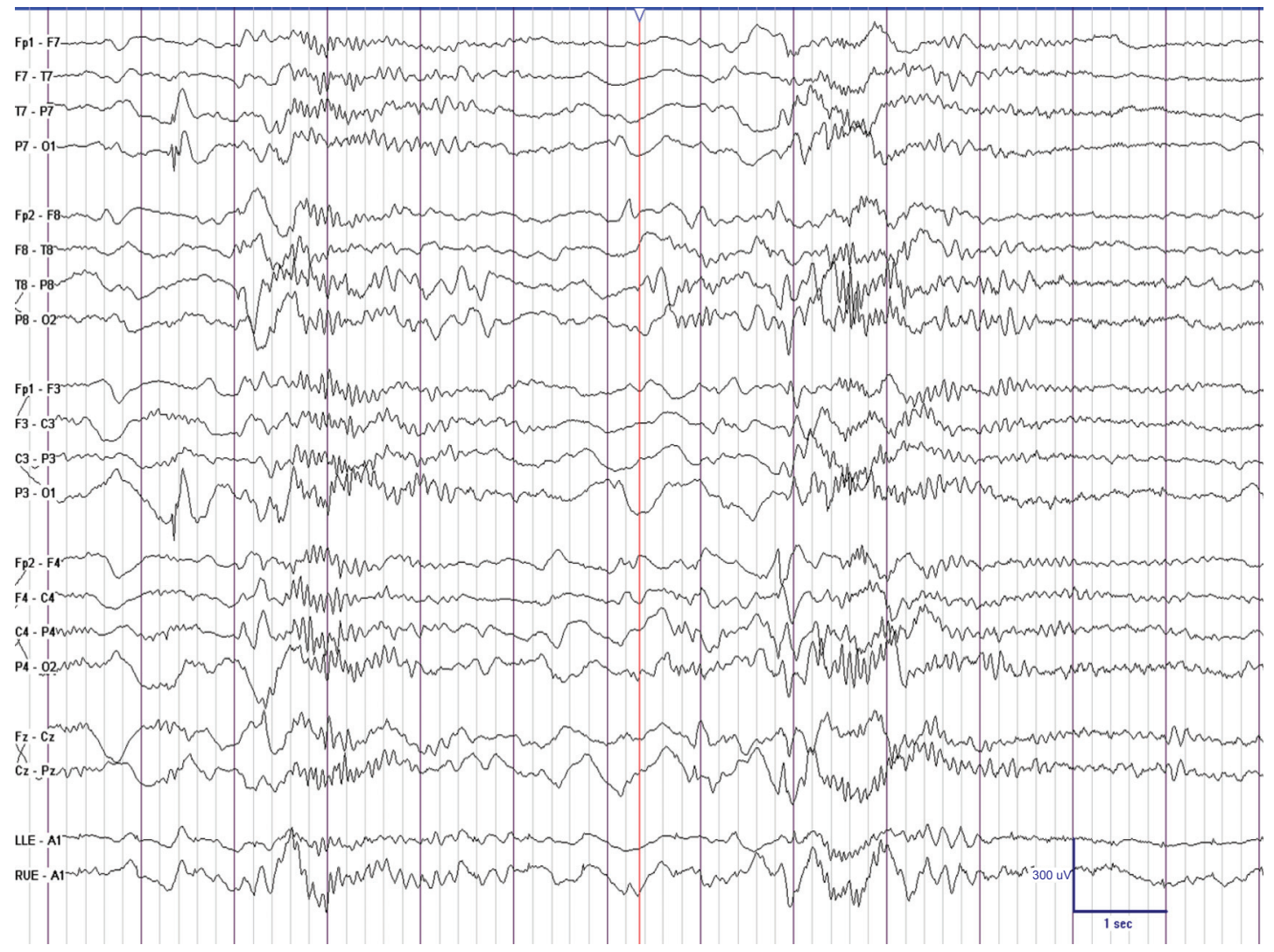

Figure 2 This is an EEG of a 8-year-old boy with Lennox-Gaustaut syndrome demonstrating paroxysmal fast activity during sleep.There were no observable clinical changes noted during the discharge. 
severity of intellectual impairment and is a poor prognosis for normal development. ${ }^{18}$

One of the hallmarks of Lennox-Gaustaut syndrome is the presence of multiple seizure types, with as many as $95 \%$ of patients having multiple seizures. ${ }^{12}$ The most characteristic seizures are tonic and are reported in over $75 \%$ of all patients with Lennox-Gaustaut syndrome under the proper recording conditions. ${ }^{12}$ Tonic seizures have a range of semiology, from global involvement of the whole body to a slight upward movement of the eyes associated with a brief change in the respiratory and heart rate. Atypical absence seizures are also common in Lennox-Gaustaut syndrome with over $75 \%$ of patients expressing this seizure type. ${ }^{15}$ The typical semiology is the progressive impairment of consciousness that is usually incomplete and recovers gradually at the end of the event. This may be difficult to recognize in a cognitively impaired child. The third most common seizure semiology are drop attacks which occur in about $33 \%$ to $66 \%$ of all patients. ${ }^{15}$ These seizures are characterized by a sudden forward or backward falls which take place in a fraction of a second. Drop attacks caused by exclusive loss of muscle tone are rare. ${ }^{23}$ Most drop attack seizures are brief motor events associated with a fall and represent either myoclonic-atonic, myoclonic, or tonic seizures. ${ }^{23,24}$ The majority of the epileptic falls in patients are the result of tonic falls. ${ }^{23,24}$ These seizures are the most difficult to treat and most dramatic to witness due to bodily injuries involving the face and head. Other type of seizures such as generalized tonic-clonic, myoclonic jerks, and focal seizures occur in Lennox-Gaustaut syndrome but are not an integral part of the syndrome.

More than $60 \%$ of patients with Lennox-Gaustaut syndrome will have a bout of status epilepticus. ${ }^{15}$ The two most common types of status epilepticus are tonic and atypical absence. Tonic status is often induced by intravenous (iv) administration of benzodiazepine. The usual scenario is a patient who is experiencing atypical absence status epileptics and is given iv benzodiazepine to stop the atypical status..$^{25,26}$ Atypical absence status epilepticus is often unrecognized due to an already cognitively impaired child who looks just a little more lethargic, irritable, or tired. The event is usually characterized by a mild clouding of consciousness that fluctuates and may last for hours to more than a week. The EEG during a status event is often difficult to discern from the interictal EEG. The slow spike-and-wave complex discharges may become more persistent or an evolution into an atypical hypsarrhythmia pattern may occur. ${ }^{27}$

The majority of patients with Lennox-Gaustaut syndrome continue to have medically refractory epilepsy, neurocognitive problems and frequent episodes of status epilepticus. Unfortunately, approximately $50 \%$ of patients continue to have the full syndrome into adulthood. Most of the remaining patients have varying severity of epilepsy and cognitive problems with institutional care the norm. Only approximately $10 \%$ will fully remit their seizures, but many of these patients remain with cognitive problems.

\section{Treatment}

The intractability of seizures to medications and multiple seizure types lead to the use of polytherapy, often at high doses. Using high doses of medications increase the likelihood of drug side effects. In this syndrome, certain medications used to treat one type of seizure may exacerbate expression of other seizure types. Both carbamazepine and phenytoin used to treat tonic and generalized tonic clonic seizure may exacerbate atypical absence and myoclonic seizures. Intravenous benzodiazepines may induce tonic status epilepticus. ${ }^{25,26}$ Although extensively used in treatment, benzodiazepines may produce undesirable side effects of drooling, incoordination and drowsiness. Together, polytherapy, restricted range of efficacious medications and side effects of drugs on the tapestry refractory seizures, make treatment unsatisfactory.

Although not tested in formalized clinical trials, valproic acid is often used as first line therapy. Three double-blind, randomized, placebo-controlled trials have shown that several of the newer AEDs, lamotrigine, felbamate, and topiramate, are efficacious as adjunctive therapy (Tables 1 and 2). ${ }^{28-30}$ Felbamate treated patients had a mean reduction in total seizure frequency of $19 \%$, compared with $4 \%$ increase with placebo and complete cessation of atonic seizures in $17.9 \%$, compared to $0 \%$ in the untreated group. ${ }^{29}$ Patients treated with lamotrigine reported a median reduction in total seizures of $32 \%$ compared with $9 \%$ increase with placebo and when subdivided into specific seizure types, there was a median reduction in drop attacks of $34 \%$ compared to $9 \%$ in the placebo group. ${ }^{28}$ Patients in the topiramate study had a median reduction of $20.6 \%$ reduction in total seizures, compared to $8.8 \%$ reduction in the placebo group and a $14.8 \%$ decrease in drop seizures, compared to a $5.1 \%$ increase in the placebo arm. ${ }^{30}$ These studies were short in duration, with maintenance phase of the study lasting 8 to 16 weeks. In reviewing these studies in a meta-analysis, a Cochrane review concluded that no single AED could be considered highly efficacious although lamotrigine, topiramate, and felbamate may be helpful as add-on treatment for patients with Lennox-Gaustaut syndrome. ${ }^{31}$ 
Table I Methodological features of randomized, double-blind controlled trials of adjunctive drug treatment for Lennox-Gaustaut syndrome ${ }^{a}$

\begin{tabular}{|c|c|c|c|c|}
\hline & Felbamate & Lamotrigine & Topiramate & Rufinamide \\
\hline Number of patients & 73 & 169 & 98 & 138 \\
\hline Age range (years) & $4-36$ & $3-25$ & $2-29$ & $4-36$ \\
\hline EEG criteria & SSW & SSW & SSW & SSW \\
\hline $\begin{array}{l}\text { Number of seizures } \\
\text { per month }\end{array}$ & 90 & 15 & 60 & $>90$ \\
\hline $\begin{array}{l}\text { Seizure frequency } \\
\text { determination }\end{array}$ & Diary ${ }^{b}$ & Diary & Diary & Diary \\
\hline AEDs & $\mathrm{I}-2$ & $\mathrm{I}-3$ & $1-2$ & $1-3$ \\
\hline \multicolumn{5}{|l|}{ Time course (weeks) } \\
\hline Baseline & 4 & 4 & 4 & 4 \\
\hline Titration & 2 & 6 & 3 & 2 \\
\hline Maintenance & 8 & 10 & 8 & 10 \\
\hline
\end{tabular}

${ }^{a}$ Only studies that showed a significant reduction in seizures are presented. Data from derived from Glauser et al ${ }^{6}$ and $\mathrm{Hancock}$ and $\mathrm{Cross}^{31}$

'This study also used video EEG.

Abbreviations: AEDs, antiseizure drugs; EEG, electroencephalogram; SSW, diffuse slow spike-and-wave complexes.

There have been other studies using non-medical modalities to investigate seizure control. In a large multicenter study of 50 patients with Lennox-Gaustaut syndrome implanted with the vagus nerve stimulator, $43 \%$ of patients had a $50 \%$ or more reduction in seizure frequency and subsequently a $56 \%$ reduction, after 1 and 3 months of use. ${ }^{32}$ Patients undergoing corpus callostomy had a $90 \%$ reduction in drop attacks in 44/52 patients followed for a mean of 40 months. ${ }^{33}$ Unfortunately, there are little comparative data with AEDs.

Against this background of seizure intractability and lack of efficacy in seizure control, a new medication has been recently approved for use in patients with Lennox-Gaustaut. Based on data from a recent published study by Glauser et al, ${ }^{6}$ the FDA has approved the medication, rufinamide, for use in this syndrome.

\section{Rufinamide}

Rufinamide is an orally active, structurally novel compound (1-[(2,6-difluorophenyl)methyl]-1 hydro-1,2,3-triazol-4 carboxamide) that is structurally distinct from other AEDs (Figure 3). ${ }^{34}$ In experimental models, rufinamide suppresses neuronal hyper-excitability by prolonging the inactivation phase of voltage-gated sodium channels. ${ }^{35}$ In several animal models, rufinamide has been shown to have broad spectrum of anticonvulsant activity. Rufinamide suppresses maximal electroshock (MES)-induced seizures in both rats and mice models, and chemically-induced seizures in mice. Rufinamide was also effective in the pentylenetetrazol (PTZ) test in mice. ${ }^{36}$ For a complete summary of the studies investigating the pharmacology of rufinamide, the reader is referred to an excellent recent review by Perucca et al. ${ }^{37}$ Another review of rufinamide, which is more clinically based, can be found in an article by Arroyo. ${ }^{38}$

\section{Pharmacokinetics}

Rufinamide is a lipophilic compound with a $\log$ p-value of 0.88 and water solubility of $40 \mathrm{mg} / \mathrm{L} .{ }^{39}$ In a study of 3 healthy volunteers, rufinamide was extensively absorbed, with $\geq 85 \%$ of the drug absorbed. The mean maximum plasma concentration $\left(\mathrm{C}_{\max }\right)$ of rufinamide was reached within 6.6 hours following a single dose of $400 \mathrm{mg}{ }^{40,41}$ Absorption is thought to be dissolution rate-limited rather than permeability rate-limited and absorption is likely to take place throughout most of the small bowel and possibly even part of the large bowel. ${ }^{37}$ The influence of food was evaluated in three studies in healthy subjects who were given single doses of rufinamide. Taking rufinamide at least 1 hour from meal times was not found to significantly affect drug plasma rufinamide concentrations. However, when rufinamide pharmacokinetics are compared after a high-fat meal and after a 14-hour fast, differences in bioavailability become clearly evident, and may have clinical relevance. After the high fat meal, $\mathrm{C}_{\max }$ increased $56 \%$ and the area under the plasma concentration curve (AUC) increased by $36 \% .{ }^{37}$ Therefore, administering rufinamide during intervals of prolonged fasting may lead to appreciably lower plasma rufinamide levels, and therefore may alter seizure protection.

Rufinamide is extensively metabolized to a carboxylic acid derivative (CGP 47292) and to the glucuronide conjugates 
Table 2 Overview of key seizure outcomes of randomized, double-blind, controlled trials of adjunctive drug treatment for Lennox-Gaustaut syndrome ${ }^{\mathrm{a}}$

\begin{tabular}{|c|c|}
\hline Study & Outcomes vs Placebo \\
\hline \multirow[t]{8}{*}{ Felbamate } & Complete cessation of seizures \\
\hline & $10.8 \%$ vs $2.7 \%(p=0.2, R R=3.9, C l=0.5-33.2)$ \\
\hline & Complete cessation of atonic seizures \\
\hline & $17.9 \%$ vs $0 \%(p=0.1, R R=5.7, C l=0.5-49.8)$ \\
\hline & Complete cessation of tonic-clonic seizures \\
\hline & $43.7 \%$ vs $7.7 \%(p=0.08, R R=5.7, C l=0.8-40.5)$ \\
\hline & Mean change in total seizure frequency \\
\hline & $19 \%$ vs $4 \%(p=0.002)$ \\
\hline \multirow[t]{9}{*}{ Lamotrigine } & Mean reduction in drop attacks \\
\hline & $34 \%$ vs $9 \%$ \\
\hline & Responder rate for drop attacks $^{\mathrm{b}}$ \\
\hline & Responder rate ${ }^{b}$ for tonic-clonic seizures \\
\hline & $43.3 \%$ vs $20.3 \%(\mathrm{RR}=2.1, \mathrm{Cl}=2.0-3.8)$ \\
\hline & Responder rate ${ }^{b}$ for total seizures \\
\hline & $33.3 \%$ vs $15.7 \%(\mathrm{RR}=2 . \mathrm{I}, \mathrm{Cl}=2.0-3.8)$ \\
\hline & Mean change in total seizure frequency \\
\hline & $32 \%$ vs $9 \%$ (increase) \\
\hline \multirow[t]{8}{*}{ Topiramate } & Mean change in drop attacks \\
\hline & $14.8 \%$ vs $5.1 \%$ (increase) $(p=0.04)$ \\
\hline & Complete cessation of drop attacks \\
\hline & $2.2 \%$ vs $0 \%(\mathrm{RR}=3.3, \mathrm{Cl}=0.1-7.8)$ \\
\hline & Responder rate for drop attacks \\
\hline & $\begin{array}{l}45.6 \% \text { vs } 20.0 \%(\mathrm{RR}=2.9, \mathrm{Cl}=0.8-10.2 \text { for } \geq 75 \% \\
\text { reduction, } \mathrm{RR}=2.0, \mathrm{Cl}=0.9-4.6 \text { for } 50 \%-74 \% \\
\text { reduction })\end{array}$ \\
\hline & Mean reduction in total seizures \\
\hline & $20.6 \%$ vs $8.8 \%(p=0.037)$ \\
\hline \multirow[t]{8}{*}{ Rufinamide } & Median reduction of tonic-atonic seizures \\
\hline & $42.5 \%$ vs I. $4 \%(\mathrm{p}<0.000 \mathrm{I})$ \\
\hline & Responder rate ${ }^{b}$ for tonic-atonic seizures \\
\hline & $42.5 \%$ vs $16.7 \%(p=0.0020)$ \\
\hline & Median reduction in total seizures \\
\hline & $32.7 \%$ vs $11.7 \%(p=0.0015)$ \\
\hline & Patients with improvement in seizure frequency \\
\hline & $53.4 \%$ vs $30.6 \%(p=0.004 I)$ \\
\hline
\end{tabular}

${ }^{a}$ Only studies that showed a significant reduction in seizures are reported. Data from Cochrane review ${ }^{31}$ and Glauser et al. ${ }^{6}$

bResponder rate defined as \% patients achieving at least $50 \%$ reduction from baseline in seizure frequency.

Abbreviations: $\mathrm{Cl}$, confidence interval; $\mathrm{RR}$, relative risk.

of the compound CGP 47292 with minimal amounts excreted unchanged in the urine and feces. Less than $7 \%$ of the dose is excreted in the urine as the acyl-glucuronide metabolite and none of this metabolite was found in the feces. ${ }^{40}$ Human liver microsome studies indicate that rufinamide is not

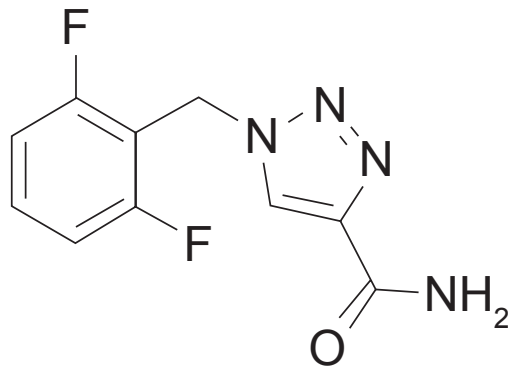

Figure 3 The molecular structure of rufinamide (I-[(2,6-difluorophenyl) methyl]-I hydro-1,2,3-triazole-4 carboxamide). Rufinamide is a triazole derivative.

metabolized via the cytochrome $\mathrm{P} 450$ system. The principal route is hydrolysis of the carboxamide by carboxylesterases into a pharmacologically inactive compound CGP 47292. The carboxylesterases are susceptible to induction by cytochrome P450 inducers. ${ }^{42}$

In a multiple-dose study in patients with epilepsy, $400 \mathrm{mg}$ given twice a day, the elimination half-life $\left(\mathrm{T}_{1 / 2}\right)$ was 6.9 hours on day 1 and 7 was unchanged on day $35 .^{37}$

There was no difference in rufinamide pharmacokinetics between older (66-77 years) and younger (18-40 years) healthy volunteers, in either single- or multiple-dosing conditions ${ }^{43}$ In a study of 16 children with epilepsy between 2 and 17 years of age treated with 10 or $30 \mathrm{mg} / \mathrm{kg}$ daily for 2 weeks, the pharmacokinetics were similar to that reported in adult patients. ${ }^{44}$ Taken together, the data suggest that rufinamide requires no age-related dose adjustments for children and elderly populations.

Due to the lack of renal excretion of unchanged drug, renal impairment has no significant influence on rufinamide pharmacokinetics. In this same study, steady state dosing was evaluated in patients undergoing hemodialysis and it was concluded that no specific dose adjustment is likely necessary for patients undergoing hemodialysis. ${ }^{37}$ No studies have examined rufinamide and patients with hepatic disease.

The extent of plasma protein binding in vitro is low, ranging from $26 \%$ to $34 \%$ at total concentrations of 0.25 to $19.6 \mu \mathrm{g} / \mathrm{mL} .{ }^{37}$ Most of the binding is to serum albumin, suggesting low potential for drug-drug interactions.

\section{Drug-drug interactions}

There have been no specific pharmacokinetic studies on rufinamide interactions with other AEDs. The effects of other AEDs on plasma rufinamide concentrations were investigated in pooled population pharmacokinetic analysis of data collected in efficacy and safety trials. ${ }^{37,45}$ Rufinamide did not alter the $\mathrm{CL} / \mathrm{F}$ of topiramate or valproic acid. At typical steady state levels of $15 \mu \mathrm{g} / \mathrm{mL}$ associated with a dose 
of 40 to $50 \mathrm{mg} / \mathrm{kg} /$ day, rufinamide increased the $\mathrm{CL} / \mathrm{F}$ of carbamazepine by $15 \%$ in children, $9 \%$ in adolescents, and $8 \%$ in adults. This predicts a reduction in carbamazepine steady state levels of $13 \%, 8 \%$, and $7 \%$, respectively. At the same rufinamide dose there was an increase in the CL/F of lamotrigine by $16 \%, 11 \%$, and $8 \%$ in children, adolescents, and adults, respectively. This would suggest a mild reduction in lamotrigine concentrations with co-administration of rufinamide. Rufinamide decreased the CL/F of both phenobarbital and phenytoin by $12 \%, 9 \%$, and $7 \%$ for phenobarbital and $17 \%, 9 \%$ and $6 \%$ for phenytoin in children, adolescents, and adults, respectively. The prediction would be an proportional increase in phenobarbital concentration, but due to non-linear elimination kinetics of phenytoin, estimations of phenytoin concentrations cannot be made with precision.

The administration of valproic acid was found to increase rufinamide concentrations. In a pooled analysis of 400 children, rufinamide concentration was increased by $60 \%$ to $70 \%$ at an average steady state concentration of valproic acid of $100 \mu \mathrm{g} / \mathrm{mL}$. At the same average steady state concentration of valproic acid, adolescents and adults has smaller increases of rufinamide concentrations, $26 \%$ and $16 \%$, respectively. In the latter two analyses, the steady state concentrations of valproic acid were 88 and $71 \mu \mathrm{g} / \mathrm{mL}$, respectively. Although the exact differences in valproic acid concentrations that would result in altering adolescent and adult concentrations of rufinamide cannot be fully ruled out, it is likely that there is an age related affect of valproic acid on rufinamide. There were no significant differences between males and females. Analysis indicated that plasma concentration of rufinamide is not significantly affected by lamotrigine, topiramate, or benzodiazepines.

In contrast to valproic acid, co-administration of carbamazepine, vigabatrin, phenytoin, phenobarbital, or primadone was found to decrease plasma rufinamide steady state concentrations. ${ }^{37}$ Again, the effect was more pronounced in children. Carbamazepine reduced plasma concentrations of rufinamide by approximately $24 \%, 19 \%$, and $21 \%$ and vigabatrin decreased concentrations by $28 \%, 18 \%$, and $14 \%$ in children, adolescents, and adults, respectively. The effects of the remaining enzyme-inducing AEDs, phenytoin, phenobarbital and primadone, were pooled. Rufinamide concentrations were reduced by $44 \%, 30 \%$, and $25 \%$ in children, adolescents and adults, respectively. The clinical relevance of these changes is unknown, but the indication is that interacting AEDs should be monitored after addition or withdrawal as well as rufinamide concentrations if the clinical situation indicates it.

\section{Safety}

The relationship between plasma rufinamide concentrations and adverse effects has been assessed in 1398 patients comprising all patients in pooled controlled studies and clinical studies. ${ }^{37,45}$ The most common side effects were dizziness $(13 \%)$, fatigue $(12 \%)$, nausea $(9 \%)$, vomiting $(7 \%)$, diplopia $(6 \%)$, and somnolence $(4 \%)$. The factors that increased the probability of adverse events were plasma concentrations of AEDs (including rufinamide), age, gender, and weight. There was an association with mildly increased side effects in adults compared to children, increased body weight, female sex, and plasma rufinamide plasma concentration.

Rufinamide side effect profile is relatively narrow and to date, non-life threatening when compared to lamotrigine, topiramate and felbamate. The use of felbamate has been restricted following reports of idiosyncratic reactions of aplastic anemia and hepatic failure. ${ }^{47}$ Lamotrigine has been found to cause potential life-threatening dermatologic reaction, especially in the context of concomitant dosing of multiple AEDs. ${ }^{48}$ Topiramate can produce oligohydrosis, hyperthermia, metabolic acidosis and glaucoma. ${ }^{49,50}$

In healthy volunteers treated with rufinamide for 18 days, the QT interval was shown to decrease in a dose-dependent manner. ${ }^{51}$ This would seem of little clinical significance. However, recently 22 patients have been described to have short QT syndrome. ${ }^{52}$ This syndrome presents with episodes of syncope, ventricular arrhythmia, and possible sudden death. Rufinamide would certainly be contraindicated in patients with this syndrome as well as other drugs that decrease the QT interval. The extent of this disorder is unknown but caution should be used in patients with history of abnormal electrocardiogram demonstrating short QT interval or family history of unexplained cardiac arrhythmia or sudden death.

\section{Rufinamide and Lennox-Gaustaut syndrome}

Lennox-Gaustaut syndrome is a catastrophic pediatric epilepsy syndrome, where over $90 \%$ of patients remain with medically refractory seizures and have severe cognitive defects and most often are institutionalized. Although some medical treatments are helpful, none have significantly influenced the treatment of this syndrome. ${ }^{31}$

As we have previously described, rufinamide is a structurally novel compound that most likely limits the excessive firing of sodium-dependent potentials. The pivotal study was a multicenter double blinded, randomized, placebo-controlled study (Tables 1 and 2$).{ }^{6}$ The requirements for entry were 
multiple seizure types which had to include atypical absence seizures and drop attacks (defined as tonic-atonic or astatic seizures), a minimum of 90 seizures the month before study entrance (the 28-day baseline period) and an EEG pattern of slow spike-and-wave complexes $(<2.5$ Hertz), and a fixed regimen of 1 to 3 concomitant AEDs. MRI or CT studies had to demonstrate that patients did not have structural criteria of a progressive lesion. The 28-day baseline period was followed by an 84-day, double-blind, placebo-controlled, parallel group treatment period. Rufinamide was dosed to a target dose of $45 \mathrm{mg} / \mathrm{kg} /$ day and this dose was reached within 7 days. Patient characteristics between the two treatment groups were similar, age range from 4 to 30 years with median age of 12 years and median weight of $34.7 \mathrm{~kg}$.

The findings were dramatic. There was an overall reduction in seizures by $32.7 \%$ per 28 days, compared to $11.7 \%$ in the placebo group $(p=0.0015)$. Moreover, there was a $42.5 \%$ decrease in drop seizures frequency, compared to the placebo increase of $1.4 \%(\mathrm{p}<0.0001)$. Due to seizure intractability to AEDs and high morbidity, the decrease in drop seizures is viewed as the primary outcome variable for treatment of Lennox-Gaustaut syndrome. ${ }^{51}$ When the results are adjusted to treatment size, rufinamide decreased drop attacks by $41.1 \%$, compared to lamotrigine reduction of $19.9 \%$ and topiramate of $14.8 \%$. The authors indicate that caution should be used, as data from cross-trial analysis with different methodologies were used to report efficacy outcomes. ${ }^{6}$

Rufinamide reduced the frequency of atypical absence seizures per 28 days ( $p<0.03$ ) and tonic seizures $(\mathrm{p}<0.02)$. No patients were seizure free during the study. Three patients developed status epilepticus, but status events are common in this syndrome, and therefore it was not clear if this was due to rufinamide dosing or the natural history of the syndrome. Although only presented in abstract form, 123 patients continued to receive medication in the open-labeled extension phase at a median dose of $1800 \mathrm{mg} /$ day (range 103-4265 mg/day) for a median duration of 432 days (range 10-1149 days). The median total seizure frequency reduction observed at 12 weeks was maintained. ${ }^{52}$ Furthermore, $21.3 \%$ of patients had $\mathrm{a} \geq 75 \%$ reduction in total seizures during the extension study. These data imply that tolerance may not occur with rufinamide use, at least over a 400-day period.

In the short double-blind study, there were few side effects, only somnolence $(24.3 \%)$ and vomiting $(21.6 \%)$ being the most cited adverse events. Treatment was also associated with lower incident of cognitive/psychiatric untoward effects (17.6\% and $23.4 \%$, respectively). In the open-labeled study, the most common adverse events were vomiting (30.6\%), pyrexia $(25.8 \%)$, upper respiratory infection (21.8\%) and somnolence (21\%). The extended study indicates that central nervous system side effects are unchanged in long term exposure of rufinamide.

\section{Conclusion}

The treatment of the catastrophic pediatric epilepsy syndrome, Lennox-Gasutaut syndrome remains unsatisfactory. Most patients remain having intolerable medically refractory seizures, cognitive delays and become institutionalized. This past year the FDA approved a new medication for adjunction treatment of this syndrome. Rufinamide is a new and structurally novel seizure medication that has proven to decrease the total frequency of seizures, in particular, the most devastating of the seizure types, drop seizures. It has few side effects and is well tolerated. It has the advantage of full therapeutic dosing within 1 week, whereas other approved medications, lamotrigine and topamax, can take up to 2 months to reach therapeutic values. There does not seem to be a change in efficacy during long-term use. It has few interactions with other seizure medications that are likely needed to help control the multiple seizure types found in this syndrome.

It is not clear if and how rufinamide will synergize with the nonmedical treatments of patients with Lennox-Gaustaut syndrome of corpus callosotomy and vagus nerve stimulation. In addition, the medical context of rufinamide with other medications that are being developed and/or other medications that have been shown to reduce seizures in small open-labeled studies should foster a better recipe for medical and surgical management of this syndrome in the future.

Taken together, the data suggest that rufinamide is a viable adjunctive treatment for patients with the LennoxGaustaut syndrome.

\section{Disclosures}

The authors disclose no conflicts of interest.

\section{References}

1. Aicardi J, Levy Gomes A. Clinical and electroencephalographic symptomatology of the 'genuine' Lennox-Gastaut syndrome and its differentiation from other forms of epilepsy of early childhood. Epilepsy Res. 1992;6:185-193.

2. Fitzgerald LF, Stone JL, Hughes JR, Melyn MA, Lansky LL. The Lennox-Gastaut syndrome: electroencephalographic characteristics, clinical correlates, and follow-up studies. Clinical Encephalogr. 1992;23(4):180-189.

3. Markand ON. Lennox-Gastaut syndrome (childhood epileptic encephalopathy). J Clin Neurophysiol. 2003;20(6):426-441.

4. Genton P, Guerrine R, Dravet C. The Lennox-Gaustaut syndrome. In: Meinardi H, editor. Handbook of Clinical Neurology: The Epilepsies, Part II. Amsterdam: Elsevier Science. 2000;73:211-222. 
5. Goldsmith IL, Zupanc ML, Buchhalter JR. Long-term seizure outcome in 74 patients with Lennox-Gastaut syndrome: effects of incorporating MRI head imaging in defining the cryptogenic subgroup. Epilepsia. 2000;41(4):395-399.

6. Glauser T, Kluger G, Sachdeo R, Krauss G, Perdomo C, Arroyo S. Rufinamide for generalized seizures associated with Lennox-Gastaut syndrome. Neurology. 2008;70(21):1950-1958.

7. Gibbs FA, Gibbs EL, Lennox WG. The influence of the blood sugar level on the wave and spike formation in petit mal epilepsy. Arch Neurol Psychiat. 1939;41:1111-1116.

8. Lennox WG, Davis JP. Clinical correlates of the fast and the slow spike-wave electroencephalogram. Pediatrics. 1950;5(4):626-644.

9. Dravet C. Encephalopathic Epileptique de l'Enfant avec Pointe-Onde Lente Diffuse [dissertation]. University of Marseille, France. 1965.

10. Gastraut H, Roger J, Soulayrol R, et al. Childhood epileptic encephalopathy with diffuse slow spike-waves (otherwise known as "petit mal variant") or Lennox syndrome. Epilepsia. 1966;7(2):139-179.

11. Ohtahara S, Ohtsuka Y, Kobayashi K. Lennox-Gastaut syndrome: a new vista. Psychiatry and Clin Neurosci. 1995;49(3):S179-S183.

12. Roger J, Dravet C, Bureau M. The Lennox-Gaustaut syndrome. Cleveland Clin J Med. 1989;56(Supp1 Pt 2):S172-S180.

13. Bauer G, Benke T, Bohr K. The Lennox-Gaustaut syndrome in adulthood. In: Niedermeyer E, Degen R, editors. The Lennox-Gaustaut Syndrome. New York: Alan R. Liss Inc; 1988. p. 317-328.

14. Guerrini R, Dravet C, Ferrari AR, Battaglia A. The evolution of epilepsy in the most common genetic forms with mental retadrdation (Down's syndrome and fragile X syndrome). Pediatr Med Chir. 1993;15(Suppl 1):19-22.

15. Beaumanoir A. The Lennox-Gastaut syndrome: a personal study. Electroencephalogr Clin Neurophysiol. 1982(35):85-99.

16. Bearmanoir A, Dravet CIeLJLp-. The Lennox-Gastaut syndrome. In: Roger J, Dureau M, Dravet C, Dreifuss FE, Perret A, Wolf P, eds. Epileptic syndromes in infancy, childhood and adolescence. 1992. 2nd ed. London: John Libbey; 1992. p. 115-132.

17. Trevathan E. Infantile spasms and Lennox-Gastaut syndrome. $J$ Child Neurol. 2002;17 Suppl 2:2S9-2S22.

18. Markand ON. Slow spike-wave activity in EEG and associated clinical features: often called 'Lennox' or "Lennox-Gastaut" syndrome. Neurology. 1977;27(8):746-757.

19. Oguni H, Hayashi K, Osawa M. Long-term prognosis of LennoxGastaut syndrome. Epilepsia. 1996;37 Suppl 3:44-47.

20. Aicardi J, Gomes AL. The Lennox-Gaustaut syndrome. Clinical and electroencephalographic features. In: Niedermeyer E, Degen R, editors. The Lennox-Gaustaut Syndrome. New York: Alan R. Liss; 1988 p. 25-46.

21. Ohtahara S, Ohtsuka Y, Yamatogi Y, et al. Early infantile epileptic encephalopathy with suppression-burst. In: Roger J, Bureau M, Dravet C, Dreifuss PE, Peret A, Wolf P, editors. Epileptic syndromes in infancy, childhood, and adolescence. 2nd ed. London: John Libbey; 1992. p. 25-34.

22. Chevrie JJ, Aicardi J. Childhood epileptic encephalopathy with slow spike-wave. A statistical study of 80 cases. Epilepsia. 1972;13(2): 259-271.

23. Ikeno T, Shigematsu H, Miyakoshi M, Ohba A, Yagi K, Seino M. An analytic study of epileptic falls. Epilepsia. 1985;26(6):612-621.

24. Egli M, Mothersill I, O'Kane M, O'Kane F. The axial spasm - the predominant type of drop seizure in patients with secondary generalized epilepsy. Epilepsia. 1985;26(5):401-415.

25. Bittencourt PR, Richens A. Anticonvulsant-induced status epilepticus in Lennox-Gastaut syndrome. Epilepsia. 1981;22(1):129-134.

26. DiMario FJ Jr, Clancy RR. Paradoxical precipitation of tonic seizures by lorazepam in a child with atypical absence seizures. Pediatr Neurol. 1988;4(4):249-251.

27. Beaumanoir A, Foletti G, Magistris M, Volanschi D. Status epilepticus in the Lennox-Gastaut syndrome. In: Niedermeyer E, Degen R, editors. The Lennox-Gaustaut Syndrome. New York: Alan R. Liss; 1988. p. 283-300.
28. Motte J, Trevathan E, Arvidsson JF, Barrera MN, Mullens EL, Manasco P. Lamotrigine for generalized seizures associated with the Lennox-Gastaut syndrome. Lamictal Lennox-Gastaut Study Group. N Engl J Med. 1997;337(25):1807-1812.

29. Efficacy of felbamate in childhood epileptic encephalopathy (LennoxGastaut syndrome). The Felbamate Study Group in Lennox-Gastaut Syndrome. N Engl J Med. 1993;328(1):29-33.

30. Sachdeo RC, Glauser TA, Ritter F, Reife R, Lim P, Pledger G. A doubleblind, randomized trial of topiramate in Lennox-Gastaut syndrome. Topiramate YL Study Group. Neurology. 1999;52(9):1882-1887.

31. Hancock E, Cross H. Treatment of Lennox-Gastaut syndrome. Cochrane Database Syst Rev. 2003;(3):CD003277.

32. Frost M, Gates J, Helmers SL, et al. Vagus nerve stimulation in children with refractory seizures associated with Lennox-Gastaut syndrome. Epilepsia. 2001;42(9):1148-1152.

33. Maehara T, Shimizu H. Surgical outcome of corpus callosotomy in patients with drop attacks. Epilepsia. 2001;42(1):67-71.

34. Bialer M, Johannessen SI, Kupferberg HJ, Levy RH, Loiseau P, Perucca E. Progress report on new antiepileptic drugs: a summary of the Fifth Eilat Conference (EILAT V). Epilepsy Res. 2001;43:11-58.

35. McLean MHJ, Schmutz M, Pozza M, et al. The influence of rufinamide on sodium currents and action potential firing in rodent neurons. [abstract]. Epilepsia. 2005;46(Suppl 8):296.

36. White HS, Schmutz M, Pozza M, Wolf H, Stables J, Kupfererg H. The anticonvulsant profile and tolerability of rufinamide in mice and rats (abstract). Epilepsia. 2005;46 Suppl 8:280.

37. Perucca E, Cloyd J, Critchley D, Fuseau E. Rufinamide: clinical pharmacokinetics and concentration-response relationships in patients with epilepsy. Epilepsia. 2008;49(7):1123-1141.

38. Arroyo S. Rufinamide. Neurotherapeutics. 2007;4:155-162.

39. Glauser T, Bialer M. Rufinamide. In: Pedley T, Engle J, editors. Epilepsy: a comprehensive textbook. 2nd ed. Philadelphia: Wolter/ Kluwer/Lippincott Williams and Wilkins; 2007. p. 1647-1654.

40. Waldmeier F, Gschwind HP, Rowan MC, et al. Metabolism of the new anticonvulsive trial drug rufinamide (CGP 33101) in healthy male volunteers (abstract). Epilepsia. 1996;37(Suppl 5):167.

41. Cheung WK, Kianifard F, Wong A, et al. Intra- and inter-subject variabilities of CGP 33101 after replicate single oral doses of two 200-mg tablets and 400-mg suspension. Pharm Res. 1995;12(12): $1878-1882$.

42. Satoh T, Hosokawa M. Structure, function and regulation of carboxylesterases. Chem Biol Inter. 2006;162(3):195-211.

43. Chung SW, Choi L, Karoplehyk MA. A pharmacokinetic evaluation of rufinamide in elderly and younger subjects [abstract]. Epilepsia. 1998;39(Suppl 6):59.

44. Sachdeo R, Rosenfeld W, Choi L, Yeah C-M, Cooper A, Karolchyk M. Pharmacokinetics and safety of adjunctive runfinamide therapy in pediatric pateints with epilepsy (abstract). Epilepsia. 1998;39(Suppl 6): $166-167$.

45. Krauss GL, Perdomo CA, Arroyo S. Short and long-term safety of rufinamide in patients with epilepsy (abstract). Epilepsia. 2005;46 (Suppl 8):205-222.

46. Fuseau E, Critchley D, Perdomo C, Arroyo S. Population pharmacokinetics drug-drug interaction analyses of rufinamide studies in patients with epilepsy. (abstract). Epilepsia. 2005;46(Suppl 8):210-211.

47. Pennell PB, Ogaily MS, Macdonald RL. Aplastic anemia in a patient receiving felbamate for complex partial seizures. Neurology. 1995;45(3 Pt 1):456-460.

48. Guberman AH, Besag FM, Brodie MJ, et al. Lamotrigine-associated rash: risk/benefit considerations in adults and children. Epilepsia. 1999;40(7):985-991.

49. Keates E, Clark T. Acute myopia and secondary angle closure glaucoma: a rare ocular syndrome in topiramate-treated patients (abstract). Neurology 2002;58:422.

50. Ben-Zeev B, Watemberg N, Augarten A, et al. Oligohydrosis and hyperthermia: pilot study of a novel topiramate adverse effect. $J$ Child Neurol. 2003;18(4):254-257. 
51. Marchand M, Critchley D, Nagy C, Faseus E. The effect of rufinamide concentration on the QT interval in healthy subjects treated during 18 days with multiple ascending doses: a population PK/PD analysis (abstract). Annual Meeting of the Population Approach Group. Vol Pamplona, Spain; 2005.

52. Bjerregaard P, Gussak I. Short QT syndrome. Ann Noninvasive Electrocardiol. 2005;10(4):436-440.

53. French JA, Kanner AM, Bautista J, et al. Efficacy and tolerability of the new antiepileptic drugs II: treatment of refractory epilepsy: report of the Therapeutics and Technology Assessment Subcommittee and Quality Standards Subcommittee of the American Academy of Neurology and the American Epilepsy Society. Neurology. 2004;62(8):1261-1273.
54. Glauser T, Kluger G, Sachdeo R, et al. Open-label extension study of the efficacy and safety of rufinamide adjunctive therapy in patients with Lennox-Gastaut syndrome (abstract). Epilepsia. 2005;46(Suppl 6):408. 